\title{
Les euphémismes dans les médias : entre voilements, démasquages et discours qui les traversent. Présentation
}

\author{
Montserrat LÓPEZ DÍAZ \\ Universidade de Santiago de Compostela \\ montserrat.lopez.diaz@usc.es \\ ORCID : 0000-0002-8637-1595
}

\author{
Annabelle SEOANE \\ Université de Lorraine \\ annabelle.seoane@univ-lorraine.fr \\ ORCID : 0000-0002-2114-7402
}

La question de l'euphémisme est abordée dans ce dossier à travers ses modes de caractérisation et de fonctionnement dans le champ médiatique ${ }^{1}$, en particulier dans la presse et les interviews politiques. Adoptant une approche sémantico-discursive et pragmatique, l'étude s'est effectuée à partir d'une collecte de données dans des corpus récents issus de plusieurs médias francophones papier et numériques.

L'euphémisme en tant que figure du discours met en jeu une non-adéquation, réelle ou ressentie, entre le signe et le référent : sollicité à la place d'une désignation attendue, il naît d'une difficulté à nommer et caractériser un référent qui, à certains égards, pose un problème. Des formulations en général imprécises ${ }^{2}$, jugées " en-deçà », sont alors utilisées pour améliorer la perception du réel conformément à l'étymologie grecque du mot euphémisme : eu 'bien' et phêmê 'parole'. Une expression neutre ou valorisante oriente ainsi le discours vers une représentation donnée comme plus favorable, du fait même qu'elle tend à estomper ses contours, en édulcorer certains aspects. Ce fonctionnement canonique de l'euphémisme a été mis en évidence depuis longtemps, tandis que d'autres dimensions de celui-ci ont été encore peu étudiées. Dans ces conditions, lorsque l'euphémisme est marqué, contesté ou commenté dans le discours, au lieu de rester voilé, il s'inscrit dans une opération de démasquage, qui va à l'encontre de son rôle initial édulcorant. Or, l'euphémisme contextualisé de

\footnotetext{
${ }^{1}$ Les articles ont été partiellement réalisés dans le cadre des projets de recherche FFI2013-42249P et FFI2017-85141P (FEDER, Ministerio de Ciencia, Innovación y Universidades, AEI).

2 Notre but n'est pas d'en faire une typologie. Pour des classements des euphémismes, on pourra se reporter à Jamet (2010 : 35-39), Horak (2010), López Díaz (2014: 50).
} 
la presse et des médias en général offre de nombreux exemples de ces deux versants en quelque sorte antagonistes.

Notre point de départ est que l'euphémisme s'ancre dans une " hétérogénéité énonciative " qui est "constitutive " ou "montrée " (Authier-Revuz, 1984), du fait qu'il est immanquablement traversé par des discours exogènes. Dans les discours journalistiques, le locuteur (le journaliste, le sujet dont les propos sont reproduits ou interprétés, ou bien un tiers) peut recourir à une énonciation euphémique socialisée et allant de soi, qui relève de l'hétérogénéité constitutive, ou mettre en cause l'utilisation même d'un euphémisme, au gré de l'hétérogénéité montrée. L'euphémisme apparaît en effet tout autant comme du déjà-dit doxique, que comme véhiculant des voix prises en charge à des degrés variables par le locuteur. C'est donc une pluralité énonciative qui émerge des différents discours traversant les euphémismes, qu'ils soient assumés ou critiqués.

Notre propos va de fait consister à appréhender cette hétérogénéité énonciative afin d'apporter un éclairage nouveau à l'euphémisme contextualisé en interrogeant les rapports que celui-ci entretient avec le dialogisme et la polyphonie. Ces deux concepts bakhtiniens ont été tantôt assimilés, tantôt dissociés, ou encore englobé l'un dans l'autre. Des chercheurs comme Moirand, Jaubert ou les auteurs du courant praxématique (Siblot, Bres, etc.) ont privilégié le concept de dialogisme, alors que Ducrot, puis Anscombre, Nølke, Donaire, notamment, ont travaillé celui de polyphonie.

Dans ce dossier, nous nous plaçons dans le sillage d'Amossy (2005), de Vion (2010) et surtout de Bonhomme (2012) en ce que nous prenons le dialogisme comme un phénomène englobant et la polyphonie comme une manifestation locale, sans qu'ils ne s'excluent nullement l'un l'autre. La polyphonie réside au niveau micro-textuel ou micro-énonciatif, dans la co-présence de voix concordantes (polyphonie convergente) ou discordantes (polyphonie divergente), les unes contribuant au voilement de l'euphémisme et les autres à son démasquage. Le dialogisme en revanche agit comme soubassement interactionnel qui s'établit au niveau global du discours en ceci que les expressions euphémiques sont porteuses d'échos préalables émanant d'autres discours où elles ont été employées et qu'elles sont également conçues en prévoyant l'éventuelle réaction du public à leur égard. À l'aune de textes de presse et d'interviews politiques notre réflexion explore ainsi les enjeux sémantiques, pragma-énonciatifs et communicationnels de l'euphémisme traversé par d'autres discours.

Les différentes contributions du dossier répondent toutes à ces prémisses et dressent dans leur ensemble un éventail qui va des expressions euphémiques tenant d'un avant-dire doxique et qui s'actualisent dans les productions linguistiques, jusqu'à d'autres qui, elles, se créent au coup par coup dans et par le discours afin de dire en principe moins que prévu. 
Pour commencer, Marc Bonhomme dans « Polyphonie divergente et mise en cause des euphémismes dans la presse écrite " prospecte, dans un cadre pragmatique, des positionnements de contestation des euphémismes dans certains sous-genres journalistiques tels les articles factuels et les éditoriaux. Selon l'auteur, le dialogisme constitue un phénomène interactionnel et global, tandis que la polyphonie se présente comme un phénomène énonciatif et local. L'euphémisme acquiert alors une dimension dialogique interdiscursive qui l'ouvre sur la circulation des discours et des dires préalables, et une autre dimension interlocutive orientée sur des interactions ultérieures possibles. Mais c'est surtout la polyphonie divergente qui est soigneusement examinée, les points de vue en désaccord, à l'instar de contestations apparaissant au fil du discours qui viennent écarter bien des fois l'effet d'atténuation qui caractérise a priori les euphémismes. Cette mise en cause du bien-fondé de ceux-ci va jusqu'à entraîner leur stigmatisation et la restitution correspondante d'une dénomination donnée pour plus juste.

Montserrat López Díaz dans "Euphémisation du discours journalistique et cadrage dialogique " s'attache à montrer comment, dans des textes journalistiques de type informatif, l'euphémisme issu d'une difficulté à nommer un référent problématique peut être exploré dans l'optique du dialogisme bakhtinien. L'expression neutre ou valorisante, étant porteuse d'échos extérieurs et antérieurs, d'empreintes des discours où le mot a été utilisé, va alors orienter le discours vers une vue plus favorable de la réalité. Sans aucun marquage graphique particulier apte à attirer l'attention sur lui, l'euphémisme est examiné dans deux perspectives dialogiques : un angle interdiscursif indexé sur une doxa plurielle, mais surtout en affinité avec la modération et la dédramatisation du discours, et un autre angle interlocutif du fait que le locuteur est censé aller à la recherche de la connivence avec autrui.

Alicja Kacprzak dans sa contribution "Le discours polyphonique sur l'obésité » propose un panorama recoupant la terminologie et le contexte de perception de l'obésité comme imperfection physique, notamment dans certains articles de presse. Ceux-ci reproduisent le discours de l'administration et de la sphère publique en général qui circulent dans la société. Représentée défavorablement, l'obésité y apparaît désignée avec des visées euphémisantes, dysphémisantes ou neutres. Les euphémismes et les dysphémismes fonctionnent comme des figures dialogiques où opère la polyphonie, qu'elle soit convergente ou divergente. La polyphonie convergente induit une prise en charge contre la doxa négative environnante, et l'euphémisme s'emploie alors à mitiger la parole, tandis que dans le cas du dysphémisme apparaît une péjoration parfois narquoise qui en amoindrit la portée dépréciative. La polyphonie divergente se repère à la prise de distance de l'énonciateur et lorsqu'il se met à produire un certain jugement : d'un côté, il peut juger l'atténuation comme non appropriée et, de l'autre, il utilise le dysphémisme pour dénoncer l'hypocrisie de la parole politiquement correcte. 
L'article suivant, "Euphémisation, conflit dénominatif et ethos dans les entretiens politiques médiatiques ", de Ruggero Druetta prolonge, par une analyse multimodale (segmentale, prosodique et mimo-gestuelle) sur un corpus oral, l'exploration de dissensus de la part de personnalités politiques interviewées. Il explique le fonctionnement d'un "dialogisme externe " qui entre en jeu sous la forme d'une négociation de la nomination des référents discursifs entre les partenaires de l'interaction, tout en mettant l'accent sur les nominations en concurrence du même objet et sur la bataille de chacun des interlocuteurs pour faire admettre son point de vue: d'une part, la formulation dysphémique du journaliste et, de l'autre, celle du politique reformulant les propos de façon euphémique et politiquement correcte, selon son appartenance idéologique. Les partenaires entrent ainsi en négociation pour imposer la nomination qu'ils jugent appropriée du référent. Le processus de rectification relève du dialogisme interdiscursif du fait qu'il régit des discours antérieurs, du dialogisme intralocutif puisqu'il s'agit de mettre en lien les prises de parole préalables du politique avec l'ethos qu'il déploie lors de l'entretien et, enfin, il relève du dialogisme interlocutif par la matérialité de l'échange intersubjectif en face à face. C'est dans ce cadre qu'évolue la négociation polyphonique des points de vue antagonistes et des positionnements argumentatifs.

Annabelle Seoane dans son article, "Euphémiser, se poser, s'opposer, s'imposer. Quelques stratégies d'atténuation autour de l'" affaire Fillon ", se focalise sur un événement discursif saillant à un moment donné dans la sphère médiatique, avec les stratégies d'évitement et de désamorçage qu'il a pu véhiculer. En réponse à un contexte dysphémique médiatique, les partisans du candidat François Fillon à la présidentielle française de 2017 se sont lancés dans des contre-discours de mitigation à visée euphémisante afin de réhabiliter le candidat. L'auteure fait apparaître une dialogisation du discours qui va dans deux directions : soit comme renvoi à un interdiscours qui atténue, soit comme remise en cause de cet interdiscours. L'euphémisme émerge alors comme résultatif, s'inscrivant entre langue et discours, alors que l'euphémisation opère dans la dynamique entre discours et interdiscours.

Pour conclure, cette présentation a pour fil conducteur le postulat que l'euphémisme ne se perçoit que dans un rapport complexe à son co(n)texte. La diversité des contributions du dossier sur la problématique de l'euphémisme permet ainsi de confronter des perspectives à travers la focale du discours qui ne peut dorénavant s'envisager comme replié sur lui-même. Il appartient ensuite à l'analyste d'en explorer les configurations et les ouvertures possibles. C'est tout l'enjeu de notre réflexion présente et à venir. 


\section{RÉFÉRENCES BIBLIOGRAPHIQUES}

Allan, Keith \& Kate BURRIDGE (2006): Forbbiden Words. Cambridge, Cambridge University Press.

AmOSsY, Ruth (2005): «De l'apport d'une distinction : dialogisme vs polyphonie dans l'analyse argumentative ", in Jacques Bres, Pierre P. Haillet, Sylvie Mellet, Henning Nølke \& Laurence Rosier (éd.), Dialogisme et polyphonie. Approches linguistiques. Bruxelles, De Boeck, 63-73.

ANSCOMBRE, Jean-Claude (2005): «Le ON-locuteur : une entité aux multiples visages ", in Jacques Bres, Pierre P. Haillet, Sylvie Mellet, Henning Nølke \& Laurence Rosier (éd.), Dialogisme et polyphonie. Approches linguistiques. Bruxelles, De Boeck, 75-94.

AUTHIER-REVUZ, Jacqueline (1984) : "Hétérogénéité(s) énonciative(s)». Langages, 73, 98-111.

BaKhtine, Mikhaïl (1970) : La Poétique de Dostoievski. Traduit du russe par I. Kolitcheff et présenté par J. Kristeva. Paris, Seuil. [éd. orig. : 1963].

BOnHOMME, Marc (2005) : Pragmatique des figures du discours. Paris, Champion.

BONHOMME, Marc (2012) : «La réception de l'euphémisme : entre réussite et échec interactif ", in Marc Bonhomme, Mariela de la Torre \& André Horak (éd.), Études pragmatico-discursives sur l'euphémisme. Frankfurt, Peter Lang, 73-88.

BRES, Jacques (2005) : "Savoir de quoi on parle : dialogue, dialogal, dialogique ; dialogisme, polyphonie... », in Jacques Bres, Pierre P. Haillet, Sylvie Mellet, Henning Nølke \& Laurence Rosier (éd.), Dialogisme et polyphonie. Approches linguistiques. Bruxelles, De Boeck, 47-61.

Charaudeau, Patrick \& Dominique Maingueneau (2002): Dictionnaire d'analyse $d u$ discours. Paris, Seuil.

DÉTRIE, Catherine, Paul SIBLOT \& Bertrand VéRINE (2001): Termes et concepts pour l'analyse du discours. Une approche praxématique. Paris, Champion.

DONAIRE, María Luisa (2014) : "Prise en charge et polyphonie, deux notions pour une théorie ». Cahiers de lexicologie, 105/2, 33-56.

DuCROT, Oswald (1980) : Les Mots du discours. Paris, Minuit.

DUCROT, Oswald (1984) : "Esquisse d'une théorie polyphonique de l'énonciation ", in Le Dire et le dit. Paris, Minuit, 171-237.

HORAK, André (2010): L'Euphémisme. Entre tradition rhétorique et perspectives nouvelles. Munich, Lincom Europa.

JAMET, Denis (2010) : "Historique et procédés linguistiques de l'euphémisme », in Denis Jamet \& Manuel Jobert (éd.), Empreintes de l'euphémisme. Tours et détours. Paris, L'Harmattan, 31-49.

JAUBERT, Anna (2008) : «Dire et plus ou moins dire. Analyse pragmatique de l'euphémisme et de la litote ». Langue française, 160, 105-116. 
LÓPEZ DÍAZ, Montserrat (2014) : "L'euphémisme, la langue de bois et le politiquement correct : changements linguistiques et stratégies énonciatives ». L'Information grammaticale, $143,47-55$.

MOIRAND, Sophie (2010) : "Retour sur une approche dialogique du discours ", in Marion Colas-Blaise, Mohamed Kara, Laurent Perrin \& André Petitjean (éd.), La Question polyphonique ou dialogique en sciences du langage. Metz, CELTED-Recherches linguistiques, 375-398.

NølKe, Henning, Kjersti FløTTUM \& Coco NORÉN (2004): ScaPoLine-La théorie scandinave de la polyphonie linguistique. Paris, Kimé.

PERRIN, Laurent (2004) : "La notion de polyphonie en linguistique et dans le champ des sciences du langage ». Questions de communication, 6, 265-282.

SiBlOT, Paul (2001) : "De la dénomination à la nomination. Les dynamiques de la signifiance nominale et le propre du nom ». Cahiers de praxématique, 36, 189-214.

VION, Robert (2010) : "Polyphonie énonciative et dialogisme", in Colloque international Dialogisme: langue, discours. Université de Montpellier. Disponible sur: https://www.praxiling.fr/spip.php?action=acceder_document\&arg=538\&cle=e618d4be7cb7 6beeee29952c23f4c299231154cc\&file=pdf_Vion1.pdf. 